\title{
Baryon electromagnetic form factors at BESIII
}

\author{
Alaa Dbeyssi ${ }^{1, \star}$ (on behalf of BESIII collaboration) \\ ${ }^{1}$ Helmholtz-Institut Mainz, Germany
}

\begin{abstract}
Electromagnetic form factors are fundamental quantities which parameterize the electric and magnetic structure of hadrons. This contribution reports on the measurements of baryon electromagnetic form factors at the BESIII experiment in Beijing. The Beijing $e^{+} e^{-}$collider BEPCII is a double-ring symmetric collider running at $\sqrt{s}$ between 2.0 and $4.6 \mathrm{GeV}$. Baryon electromagnetic form factors can be measured at BESIII in direct $e^{+} e^{-}$-annihilation and in initial state radiation processes. Based on the data collected by the BESIII detector at 12 center of mass energies between 2.23 and $3.67 \mathrm{GeV}$, the $e^{+} e^{-} \rightarrow \bar{p} p$ cross section and the time-like proton form factor is measured. Preliminary results from the analysis of the initial state radiation process $e^{+} e^{-} \rightarrow \bar{p} p \gamma$ using a data set of $7.408 \mathrm{fb}^{-1}$ collected at center-of-mass energies between 3.773 and $4.6 \mathrm{GeV}$, are also presented. The cross section for $e^{+} e^{-} \rightarrow \bar{\Lambda} \Lambda$ is measured based on $40.5 \mathrm{pb}^{-1}$ data collected at 4 energy points from the threshold up to $3.08 \mathrm{GeV}$. Preliminary results on the total cross section and the $\Lambda$ effective form factor are shown. Ongoing analysis based on the high luminosity energy scan from 2015 and from radiative return at different $\sqrt{s}$ are also described.
\end{abstract}

\section{Introduction}

Electromagnetic form factors (FFs) describe the modifications of the point-like photon-hadron vertex due to the structure of the hadrons. They provide information on the intrinsic electric and magnetic distributions of hadrons. Electromagnetic FFs have been associated in the Time-Like (TL) region with the time evolution of these distributions [1]. The number of electromagnetic FFs is determined by the spin of the hadron taking into account the symmetry properties of the electromagnetic interaction under the charge, parity, and time transformations. The structure of a non point-like particle of spin $S$ is parameterized in terms of $(2 S+1)$ FFs.

The electromagnetic FFs of baryons, spin 1/2 particles, can be measured in TL region through the annihilation reactions $e^{+} e^{-} \leftrightarrow \bar{B} B$ using the energy scan technique, in which the center of mass energy of the collider $(\sqrt{s})$ is varied systematically, and at each energy point a measurement of the associated cross section can be carried out. In the Born approximation, the differential cross section for the annihilation reaction $e^{+} e^{-} \rightarrow \bar{B} B$ in the centre of mass system, is expressed as a function of

\footnotetext{
^e-mail: adbeyssi@uni-mainz.de
} 
the baryon electromagnetic FFs $\left|G_{E}\right|$ and $\left|G_{M}\right|$ as [2]:

$$
\begin{aligned}
\frac{d \sigma}{d \cos \theta} & =\frac{\pi \alpha^{2} \beta C}{2 q^{2}}\left[\left(1+\cos ^{2} \theta\right)\left|G_{M}\right|^{2}+\frac{1}{\tau} \sin ^{2} \theta\left|G_{E}\right|^{2}\right], \\
\beta & =\sqrt{1-\frac{1}{\tau}}, \tau=\frac{q^{2}}{4 M^{2}}, C=\frac{y}{(1-\exp (-y))}, y=\frac{\alpha \pi}{\beta},
\end{aligned}
$$

where $\alpha$ is the electromagnetic coupling constant, and $\theta(M)$ is the scattering angle (mass) of the baryon. The Coulomb factor $C$ makes the cross section non zero at threshold [3]. It can be expressed as a product between an enhancement factor $\mathcal{E}=\frac{\alpha \pi}{\beta}$ and a resummation term $\mathcal{R}$, i. e., the so called Sommerfeld-Schwinger-Sakharaov rescattering formula. In the limit $\beta \rightarrow 0$, the Coulomb factor behaves like the enhancement factor $\mathcal{E}$ so that, the factor $\beta$ is cancelled and the cross section at threshold becomes finite. Equation 1 assumes that the interaction between baryons and leptons occurs through the exchange of one virtual photon, which carries a momentum transfer squared $q^{2}$.

The total cross section, integrated over the full range of the polar angle $\theta$ is:

$$
\sigma\left(q^{2}\right)=\frac{2 \pi \alpha^{2} \beta C}{3 q^{2} \tau}\left(2 \tau\left|G_{M}\right|^{2}+\left|G_{E}\right|^{2}\right) .
$$

The measurement of the differential cross section (1) at a fixed energy allows the determination of the FF ratio $R=\left|G_{E}\right| /\left|G_{M}\right|$. With a precise knowledge of the normalization and the luminosity, a separate determination of $\left|G_{E}\right|$ and $\left|G_{M}\right|$ is possible. The quantity usually measured when the angular distributions cannot be precisely studied (due to the low statistics) is the effective FF, introduced as a linear combination of $\left|G_{E}\right|^{2}$ and $\left|G_{M}\right|^{2}$ :

$$
\left|G_{e f f}\right|=\sqrt{\frac{2 \tau\left|G_{M}\right|^{2}+\left|G_{E}\right|^{2}}{2 \tau+1}} .
$$

The initial state radiation (ISR) technique is an effective tool to measure hadronic cross section at high luminosity $e^{+} e^{-}$-storage rings, such as the Beijing Electron-Positron Collider II (BEPCII). Due to the fact that annihilation of $e^{+} e^{-}$is mostly accompanied by emission of one or several photons from the initial state, the high luminosity collected at the $e^{+} e^{-}$collider experiments, allows for a complementary approach to the energy scan technique in hadron cross section measurements: events with ISR photons lead to a reduction of the invariant mass $\left(q^{2}\right)$ of the proton-antiproton system, and hence allow for a measurement of the baryonic FFs over a wide range of $q^{2}$ below $\sqrt{s}$.

\section{BESIII detector and data sets}

The BESIII detector [4] is a general purpose spectrometer located at BEPCII. BEPCII is a double ring $e^{+} e^{-}$collider running at center of mass energies $(\sqrt{s})$ between 2.0 and $4.6 \mathrm{GeV}$ and reached a peak luminosity of $1.0 \times 10^{33} \mathrm{~cm}^{-2} \mathrm{~s}^{-1}$ at $\sqrt{s}=3770 \mathrm{MeV}$. The cylindrical BESIII detector has an effective geometrical acceptance of $93 \%$ of $4 \pi$. It contains a small cell, helium-based $\left(60 \% \mathrm{He}, 40 \% \mathrm{C}_{3} \mathrm{H}_{8}\right)$ main drift chamber (MDC) which provides momentum measurements of charged particles with a resolution of $0.5 \%$ at $1 \mathrm{GeV} / \mathrm{c}$ in a 1 Tesla magnetic field. The energy loss $(\mathrm{dE} / \mathrm{dx})$ measurement provided by the MDC has a resolution better than 6\%. A time-of-flight system (TOF) consisting of 5-cm-thick plastic scintillators with a time resolution of $80 \mathrm{pb}$ in the barrel and $110 \mathrm{ps}$ in the end caps. An electromagnetic calorimeter (EMC) consisting of $6240 \mathrm{CsI}$ (Tl) in a cylindrical structure and two end caps is used to measure the energies of photons and electrons. The energy resolution of EMC (for $1 \mathrm{GeV}$ electrons and photons) is $2.5 \%$ in the barrel and $5.0 \%$ in the end caps. The position resolution 
of EMC is $6 \mathrm{~mm}$ in the barrel and $9 \mathrm{~mm}$ in the end caps for $1 \mathrm{GeV}$ electrons and photons. A muon system (MUC) consisting of $1000 \mathrm{~m}^{2}$ of Resistive Plate Chambers (RPC) is used to identify muons and provides a spatial resolution better than $2 \mathrm{~cm}$.

BESIII provided the world's largest samples of $e^{+} e^{-}$collisions in the $\tau$-charm region. Data samples have been accumulated at different charmonium and X, Y, Z states, and high luminosity scan experiments have been performed in the region between 2.0 and $4.6 \mathrm{GeV}$. The data collected at BESIII allows the measurement of baryon FFs using the direct $e^{+} e^{-}$annihilations and the initial state radiation processes.

\section{Proton form factors at BESIII}

\subsection{Measurement of the $e^{+} e^{-} \rightarrow \bar{p} p$ using the scan technique}

Using the $157 \mathrm{pb}^{-1}$ data samples collected in 2011 and 2012, BESIII measured the cross section of $e^{+} e^{-} \rightarrow \bar{p} p$ at 12 center of mass energies between 2.2 and $3.7 \mathrm{GeV}$ [5]. The event selection criteria, the estimation of the background contamination and the evaluation of the signal efficiencies were obtained based on the Monte Carlo simulations for the signal and the different background processes. The Born cross section of $e^{+} e^{-} \rightarrow \bar{p} p$ is calculated by:

$$
\sigma_{\text {Born }}=\frac{N_{o b s}-N_{b k g}}{L \epsilon(1+\delta)},
$$

where $N_{o b s}$ is the number of selected events, $N_{b k g}$ is the estimated number of background events, $L$ is the integrated luminosity determined at each center of mass energy, $\epsilon$ is the detection efficiency of the signal and $(1+\delta)$ is the radiative correction factor calculated using the CONEXC event generator [6]. The corresponding effective electromagnetic FF of the proton is deduced from the measured cross section using 2 and 3 . The results on the Born cross section and the proton effective FF are shown in figure 1 together with the previous experimental measurements. Below $\sqrt{s}=3.08 \mathrm{GeV}$, BESIII improves the precision on the Born cross section by $30 \%$ compared to the most precise data measured by BaBar [7, 8]. The ratio of the proton FFs is extracted from the fit to the distribution of the proton polar angle using the large data samples collected at $\sqrt{s}=2232.4,2400.0$ and a combined sample at $\sqrt{s}=3050.0,3060.0$ and $3080.0 \mathrm{MeV}$. The measured ratios of the proton FFs are close to unity and they are consistent with the BABAR results in the same $q^{2}$ region.

\subsection{Measurement of the $e^{+} e^{-} \rightarrow \bar{p} p$ using the ISR technique}

Based on $7.408 \mathrm{fb}^{-1}$ data samples collected with the BESIII detector at center of mass energies between 3.773 and $4.600 \mathrm{GeV}$, the proton FFs have been measured using the initial state radiation technique. In this work, the two charged tracks (proton and antiproton) are reconstructed with the MDC, and the ISR photon is assigned to the highest energy photon detected by the EMC. Only the events where the ISR photons are emitted within the acceptance of the EMC are selected (tagged ISR analysis). The ratio of the proton FFs has been extracted in six intervals of $\bar{p} p$-invariant mass from the threshold up to $3.0 \mathrm{GeV} / \mathrm{c}^{2}$. The Born cross section of $e^{+} e^{-} \rightarrow \bar{p} p$ and the proton effective $\mathrm{FF}$ have been also measured in 31 intervals of $\bar{p} p$-invariant mass below $3.0 \mathrm{GeV} / \mathrm{c}^{2}$.

Figures 2 and 3 show the preliminary results for the Born cross section, the proton effective FF and the proton FF ratio, respectively. The results are consistent with the previous experiments. The total uncertainty, dominated by the statistical one, is between $20 \%$ and $35 \%$ for the ratio and between $10 \%$ and $64 \%$ for the Born cross section. 

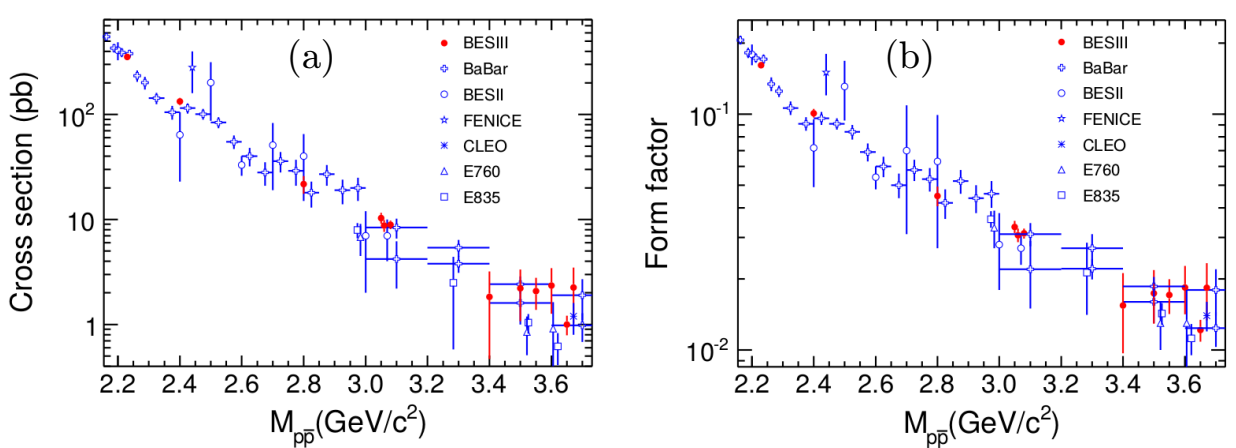

Figure 1. Comparison of the Born cross section of $e^{+} e^{-} \rightarrow \bar{p} p$ (a) and the proton effective FF (b) between BESIII (red points) and previous measurements [5].
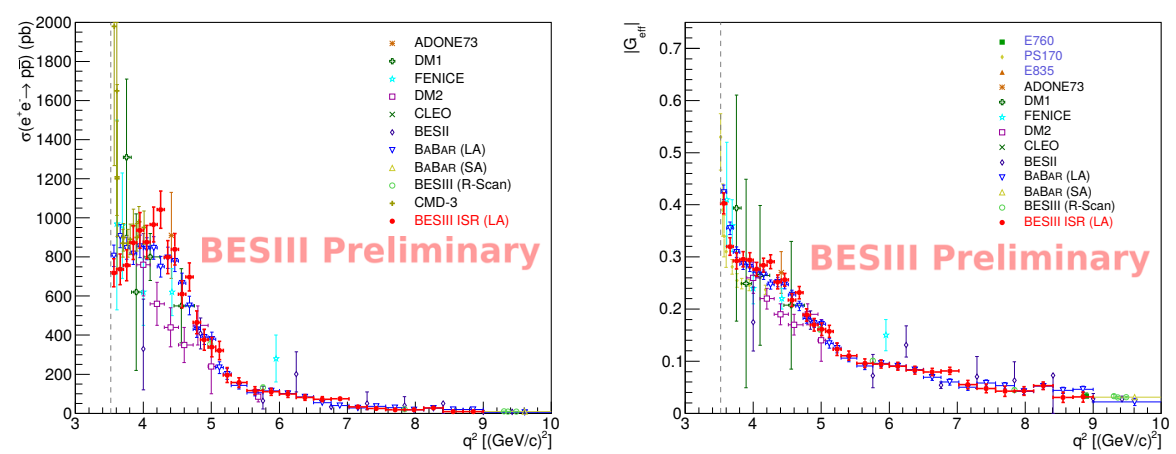

Figure 2. BESIII preliminary results on the Born cross section of $e^{+} e^{-} \rightarrow \bar{p} p$ (a) and the effective proton (b) from the tagged ISR analysis (BESIII ISR (LA), red points).

The angular distribution of ISR photon in $e^{+} e^{-} \rightarrow p \bar{p} \gamma$ is shown in figure 4 . The differential cross section of $e^{+} e^{-} \rightarrow p \bar{p} \gamma$ reaches the maximum values at $\cos \theta_{\gamma} \rightarrow \pm 1\left(\theta_{\gamma} \sim 0^{\circ}, 180^{\circ}\right)$. These regions are not covered by the EMC. The $e^{+} e^{-} \rightarrow p \bar{p} \gamma$ events in which the ISR photons cannot be detected have been also analysed (untagged ISR analysis). The measurement of these events benefits from the fact that the number of statistics is increased by a factor $\sim 3$ compared to the tagged analysis. The difficulty of this measurement relays on the signal/background separation using only the information on two detected particles, proton and anti-proton. Signal and background processes have been simulated with the BESIII Offline Software System (BOSS) [9] which includes full detector simulation and event reconstruction. The background channels are almost suppressed by the event selection criteria keeping the signal efficiency around $16 \%$. The same data samples $\left(7.408 \mathrm{fb}^{-1}\right)$ used for the tagged analysis have been analysed and the signal events in which the ISR photon is not detected have been selected. The results are not yet released by the BESIII collaboration. Based on the Monte Carlo simulations, using PHOKHARA 9.1 event generator [10], the Born cross section of $e^{+} e^{-} \rightarrow p \bar{p}$ can 


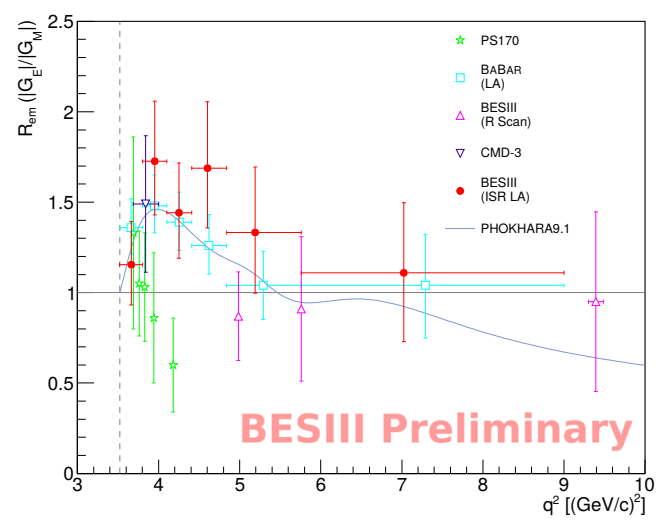

Figure 3. BESIII preliminary results on the ratio of the proton FFs from the tagged ISR measurement of $e^{+} e^{-} \rightarrow$ $\bar{p} p \gamma$.

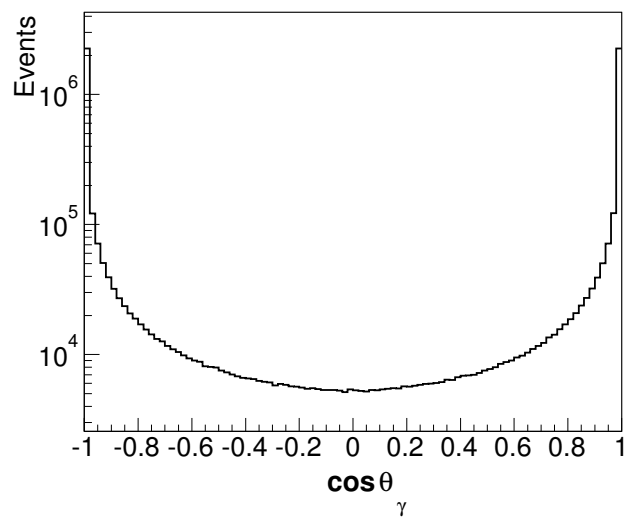

Figure 4. Angular distribution of the ISR photons $\left(\cos \theta_{\gamma}\right)$ in $e^{+} e^{-} \rightarrow p \bar{p} \gamma$ at $\sqrt{s}=4.23 \mathrm{GeV}$ (simulated with PHOKHARA 9.1 event generator).

be measured in the region between 2 and $\sim 4 \mathrm{GeV} / \mathrm{c}^{2}$, with a statistical accuracy between $5 \%$ and $35 \%$.

\section{Hyperon form factors at BESIII}

BESIII released recently preliminary results on the measurement of the $e^{+} e^{-} \rightarrow \bar{\Lambda} \Lambda$ cross section. Data samples of $40.5 \mathrm{pb}^{-1}$ collected at $\sqrt{s}=2.2324,2.400,2.800$ and $3.080 \mathrm{GeV}$ have been analysed. The lowest energy point, $2.2324 \mathrm{GeV}$, is only $1.0 \mathrm{MeV}$ above the threshold of the $\bar{\Lambda} \Lambda$ production. At this energy, the $e^{+} e^{-} \rightarrow \bar{\Lambda} \Lambda$ process is analysed from two decay modes, $\bar{p} p \pi^{+} \pi^{-}$and the inclusive $\bar{n} \pi^{0}+X$. The measurement of the cross section of $e^{+} e^{-} \rightarrow \bar{\Lambda} \Lambda$ process from the two decay modes gives 

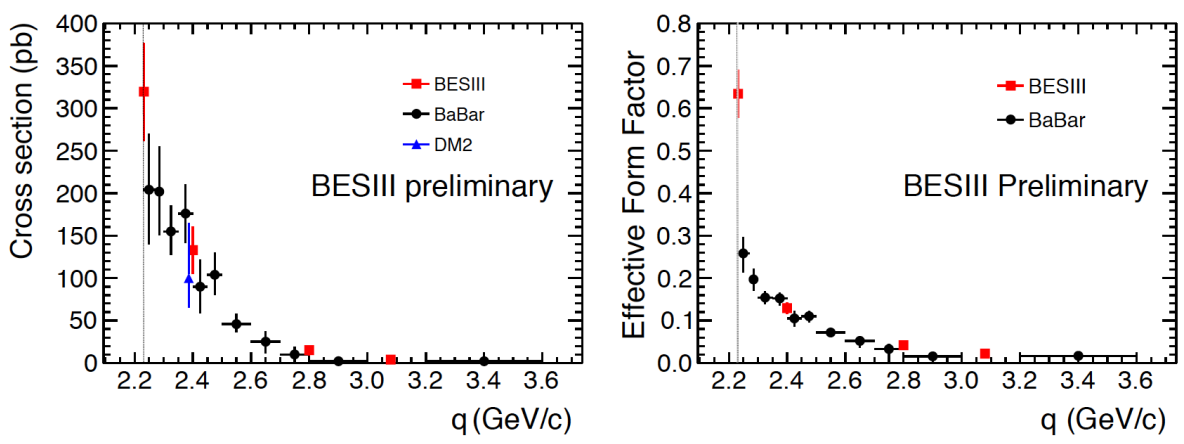

Figure 5. BESIII preliminary results (red points) on the Born cross section of $e^{+} e^{-} \rightarrow \bar{\Lambda} \Lambda$ (a) and the $\Lambda$ effective FF (b).

consistent results. At $\sqrt{s}=2.400,2.800$ and $3.080 \mathrm{GeV}$, the cross section is measured using only the charged decay mode $\left(\bar{p} p \pi^{+} \pi^{-}\right)$. The preliminary results on the Born cross section and the hyperon effective FF are shown in figure 5. At $\sqrt{s}=2.2324 \mathrm{GeV}$, the average cross section is $318 \pm 60 \mathrm{pb}$, which is significantly larger than the expected value assuming no Coulomb interaction between the produced neutral hyperons. At the higher energy points, the results are consistent with the previous data.

\section{Summary and prospects}

The experimental measurements of the TL baryon FFs close to the threshold region shows some unexpected features $[11,12]$. In this context, a precise and complete measurement of the baryon FFs in this region is of great importance. BESIII is an excellent laboratory for the measurement of baryon FFs, since both ISR and scan methods can be performed, and the kinematical threshold for the different baryon pair production is covered by the available data samples.

In 2015, BESIII performed high luminosity scan in 22 energy points between 2.0 and $3.08 \mathrm{GeV}$. Based on these data samples, the proton electromagnetic FFs $\left|G_{E}\right|$ and $\left|G_{M}\right|$ can be extracted separately. The expected precision on the ratio of the proton FFs is at the level of $10 \%$. In addition to the proton, BESIII can provide the most precise measurements of the neutron FFs with the $e^{+} e^{-} \rightarrow \bar{n} n$ and $e^{+} e^{-} \rightarrow \bar{n} n \gamma$ processes. The determination of $\Lambda \mathrm{FFs},\left|G_{E}\right|,\left|G_{M}\right|$ and the relative phase between them is also possible. The polarization of $\Lambda$ in $e^{+} e^{-} \rightarrow \bar{\Lambda} \Lambda$, which contains information on the relative phase between $\left|G_{E}\right|$ and $\left|G_{M}\right|$, can be experimentally accessed thanks to the self-analyzing weak decay of $\Lambda$, i.e $\Lambda \rightarrow p \pi^{-}$. The measurement of the hyperons FFs at BESIII can be extended to other channels, i.e. $e^{+} e^{-} \rightarrow \bar{\Lambda}_{\bar{c}} \Lambda_{c^{+}}, \Lambda \bar{\Sigma}^{0}, \ldots$

\section{References}

[1] E. A. Kuraev, E. Tomasi-Gustafsson, and A. Dbeyssi. Phys. Lett. B 712, 240-244 (2012)

[2] A. Zichichi, S. M. Berman, N. Cabibbo, R Gatto, Nuovo Cim. 24, 170 (1962)

[3] C. Tzara, Nucl. Phys. B 18, 216-252 (1970)

[4] M. Abilikim et al. (BESIII Collaboration), Nucl. Instru. Meth. A 614, 345 (2010)

[5] M. Abilikim et al. (BESIII Collaboration), Phy. Rev. D 91, 112004 (2015) 
[6] R. G. Ping, Chin. Phys. C 38, 083001 (2014)

[7] J. P. Lees et al. (BABAR Collaboration), Phys. Rev. D 87, 092005 (2013)

[8] J. P. Lees et al. (BABAR Collaboration), Phys. Rev. D 88, 072009 (2013)

[9] Z. Y. Deng et al., HEP \& NP 30, 371 (2006)

[10] H. Czyt, M. Gunia and J. H. Kuehn, JHEP 1308, 110 (2013)

[11] A. Bianconi and E. Tomasi-Gustafsson. Phys. Rev. C 93, 035201 (2016)

[12] J. Haidenbauer and U. G. Meissner, Phys. Lett. B 761, 456 (2016) 